\title{
Relações entre Impacto do Treinamento no Trabalho e Estratégia Empresarial: o Caso da Eletronorte
}

\author{
Maria Ednei da Silva
}

\begin{abstract}
RESUMO
A pesquisa avaliou a relação entre as variáveis: Relevância do Treinamento para os Objetivos Estratégicos da Organização, Suporte à Transferência de Treinamento e Impacto de Treinamento no Trabalho. Dois grupos de treinamentos foram pesquisados em uma organização de grande porte: o primeiro, destinava-se a desenvolver competências individuais similares às competências corporativas requeridas pelos objetivos estratégicos da organização e o segundo destinava-se a desenvolver competências individuais, não similares àquelas competências. Para medir a Relevância do Treinamento para os Objetivos Estratégicos foram realizadas entrevistas com uma amostra de 33 gestores. Para medir Suporte à Transferência e Impacto do Treinamento no Trabalho utilizaramse as escalas validadas por Abbad-Oc (1999) e por Abbad e Sallorenzo (2001), numa amostra de 375 empregados treinados. Encontraram-se diferenças significativas nas variáveis pesquisadas entre os dois grupos sendo que Impacto de Treinamento no Trabalho foi maior no primeiro grupo de treinamento. Suporte Gerencial e Social à Transferência confirmou-se como o maior preditor de Impacto de Treinamento no Trabalho.
\end{abstract}

Palavras-chave: treinamento; impacto do treinamento no trabalho; recursos humanos; estratégia organizacional.

\begin{abstract}
The research evaluated the relation among the variables: Relevance of the Training for the Organization's Strategic Goals, Transference Support of Training and Training Impact at Work. Two groups of training were researched at the big organization: the first, was destined to development individual competencies taught that were similar to the corporative competences required by the organization strategic goals, and the second was destined to development individual competencies that were not similar to those competences. The Relevance of the Training for the Organization's Strategic Goals was measured by the interviews with the sample of 33 managers. Transference Support of Training and Training Impact at Work it was measured by a scale validated by Abbad-Oc (1999) and by Abbad \& Sallorenzo (2001), with the sample was constituted of 375 trained employees. The results verified that there were significant differences in the variables between both groups and it was found that the variable Training Impact at work it is bigger in the first group of training. The Managerial and Social Support to the Transfer is the best predictors of for the variable Training Impact at Work.
\end{abstract}

Key words: training; training impact at work; human resource; organizational strategy. 


\section{INTRODUÇÃO}

As profundas mudanças socioculturais, políticas, tecnológicas e econômicas que caracterizam o cenário atual das organizações, exigem qualificação da mão-de-obra para dominar tecnologias sofisticadas e atuar em ambiente competitivo. Mais do que nunca, as organizações estão procurando aumentar a competitividade dos produtos e serviços. Com tal objetivo, têm investido maciçamente em programas de treinamento dos empregados, como forma de aumentar as competências individuais com vistas às core competencies da organização. Segundo Green (1999, p. 5), "Competência individual é um conjunto de hábitos de trabalho mensuráveis e habilidades pessoais necessárias para se alcançar um objetivo de trabalho", o autor colocou ainda que "Core Competencies são os conhecimentos e habilidades técnicas únicas que têm impacto no produto e no serviço da organização e que lhe dão vantagem competitiva no mercado" (p. 6). Se as competências individuais desenvolvidas forem relevantes para os objetivos estratégicos organizacionais, espera-se maior impacto do treinamento no trabalho e, por consequiência, nas core competencies da organização.

É alto o volume do investimento em programas de treinamento e, em um ambiente em que os recursos são cada vez mais escassos, torna-se vital o alinhamento dos objetivos de treinamento com os objetivos estratégicos da organização. A contribuição do treinamento para os objetivos estratégicos organizacionais pode ser um resultado empresarial consistente, porque, de acordo com Tannenbaum e Yukl (1992, p. 401): "treinamentos deveriam dar suporte à direção estratégica da organização, e os objetivos de treinamento deveriam ser alinhados com os objetivos organizacionais". Para Kozlowski, Brown, Weissbein, Cannon-Bowers, e Salas (2000, p. 158), a razão para o investimento em treinamento é a necessidade de aumentar as habilidades dos empregados para se alcançarem os objetivos estratégicos da organização. Segundo esses autores, pressões por melhorias contínuas e adaptabilidade organizacional começam a enfatizar a presumível ligação entre os resultados de treinamento e a eficácia da organização.

As organizações vêm solicitando a avaliação do retorno do investimento em treinamento. Para Salas e Cannon-Bowers (2001, p. 472), é crescente a inquietação por parte das organizações quanto à certeza do investimento em treinamento ser justificado pela performance organizacional, como aumento da produtividade, lucros ou incremento das fatias de mercado. Conforme esses autores, as organizações estão começando a questionar o valor agregado do 
capital investido em treinamento, o que levará fatalmente à avaliação da sua eficácia para os resultados organizacionais.

A convergência dos objetivos de treinamento com os da estratégia organizacional tem sido conclamada na literatura como condição para a eficácia dessa intervenção para os resultados organizacionais (Kozlowski et al., 2000; Salas \& Cannon-Bowers, 2001; Tannenbaum \& Yukl, 1992). A eficácia de um treinamento passa pela transferência das habilidades adquiridas para o trabalho do indivíduo na organização. Partindo desse pressuposto, este estudo investigou a relação entre a relevância do treinamento para os objetivos estratégicos da organização, o suporte à transferência do treinamento e o impacto do treinamento no trabalho.

\section{Treinamento e Estratégia Organizacional: Vantagem Competitiva da Organização}

A vantagem competitiva da organização é creditada, em algum grau, à integração dos programas de treinamento com a estratégia organizacional. Porter (1989, p. 22) afirma que a liderança empresarial num determinado mercado, por si só, não indica competitividade, mas a vantagem competitiva, que tem sua origem nas inúmeras atividades desenvolvidas pela empresa e é afetada pelo treinamento dos empregados. Para Hill e Jones (1998, p. 3), os resultados dos programas de treinamento adotados pela empresa afetam a produtividade dos empregados, sendo esta um determinante-chave para a eficiência empresarial e a redução de custos.

Segundo C. A. Lengnick-Hall e M. L. Lengnick-Hall (1990, p. 48), pela expansão da gestão de recursos humanos além das fronteiras da organização, o impacto potencial dos programas afetos à área nos processos estratégicos da empresa será maior. Para esses autores, uma organização que desenvolva programas de treinamento somente para os próprios empregados não ganha vantagem competitiva; mas quando aumenta os conhecimentos e as habilidades dos componentes de toda a cadeia de valor (C. A. Lengnick-Hall \& M. L. LengnickHall, 1990, p. 50). Treinamento tem, portanto, papel fundamental para a competitividade organizacional, quando capacita os empregados e toda a cadeia de valor nos processos estratégicos da organização.

Empregados treinados constituem verdadeira fonte de vantagem competitiva, uma vez que são os responsáveis, em última análise, pela inserção estratégica do produto ou serviço da organização no mercado. Se uma empresa tem como 
objetivo estratégico aumentar a produtividade, antes dos programas de avaliação e recompensa, terá de se assegurar de que os empregados possuem as competências individuais necessárias para o trabalho, tendo em vista aquele objetivo.

Uma abordagem corrente sobre estratégia organizacional é a da core competence, que presume o desenvolvimento de vantagens competitivas peculiares às organizações, difíceis ou até mesmo impossíveis de serem imitadas. Segundo Hamel e Prahalad (1995, p. 263), para administrar o estoque de competências específicas, a empresa precisa ser capaz de desmembrar essas competências até o nível de indivíduos com talentos específicos, e sugerem que cada funcionário seja o detentor das competências da organização. Para esses autores muitas empresas não possuem um processo de alocação do talento que constitui a core competence, a exemplo do que ocorre com o capital, sendo a diferença entre o valor dos ativos e o valor contábil das empresas definida pela sua core competence (Hamel \& Prahalad, 1995, p. 270). Ulrich (1998, p. 82), por sua vez, afirma que, em organizações de sucesso, as competências individuais estão se transformando em capacidades organizacionais, ou seja, a core competence determina o valor de uma empresa e as competências individuais dos empregados têm peso nesse valor.

Legge (1995, p. 104) sugere programas de treinamento ajustados ao estágio de desenvolvimento da empresa e ao nível da orientação estratégica de negócios da organização. Tannenbaum e Yukl (1992, p. 401) recomendam programas de treinamento diferentes para uma organização que está fazendo downsizing das que estão em expansão, que desenvolvem novos produtos ou implementam programas de aumento de qualidade. Isso significa que os programas de treinamento guardam coerência com a estratégia da organização.

Para Latham (1988, p. 549) objetivos de treinamento estabelecem uma relação estreita com a estratégia corporativa da organização, de forma que são monitorados e sujeitos à estratégia organizacional. Também Campbell (1988, p. 190) afirma que os objetivos organizacionais determinam os objetivos de treinamento, a fim de garantir que sejam projetados para melhorar a performance dos empregados em um fator crítico de trabalho. Para tanto, requer-se alinhamento entre o que a organização necessita desenvolver no nível da estratégia corporativa e os programas de treinamento realizados.

Outra abordagem atualmente utilizada pelas organizações na formulação da estratégia organizacional é a "visão da empresa baseada em recursos", que propõe serem os recursos da empresa os principais determinantes de sua competitividade, contrapondo-se à abordagem da análise da indústria, na qual a competitividade 
da empresa é determinada pela sua posição na indústria (Oliveira Jr., 2001, p. 123). Nessa abordagem, recursos compreendem os ativos (tangíveis ou intangíveis) da empresa; e a qualificação dos empregados têm peso nos ativos intangíveis como fonte de vantagem competitiva da organização. Essa abordagem também aponta para programas de treinamento vinculados à estratégia organizacional.

Kamoche (1996, p. 214) afirma que na "visão da empresa baseada em recursos" a organização é vista como um conjunto de recursos e capacidades, tangíveis e intangíveis, requeridos para os seus produtos. Segundo esse autor, recursos são as forças e fraquezas de uma empresa que incluem os ativos tangíveis e intangíveis, como as competências básicas da organização e a aprendizagem coletiva embutida na core competence da empresa e capacidades compreendem tudo aquilo que ela pode fazer com seus recursos. Essa visão embute a concepção do alinhamento entre as competências individuais dos empregados com a core competence da empresa.

\section{Treinamento, Desenvolvimento e Educação}

Segundo a literatura científica treinamento, desenvolvimento e educação têm objetivos diferentes. Nadler (1984, p. 16) conceitua treinamento como a aprendizagem relacionada ao trabalho do indivíduo, educação como a qualificação do empregado para ocupar diferente posto de trabalho na organização e desenvolvimento como a aprendizagem destinada ao crescimento do indivíduo, não relacionada especificamente ao trabalho atual ou futuro. Bastos (1991, p. 88) afirma que a distinção entre educação e treinamento reporta-se ao nível de generalidade; a educação seria geral e se destinaria ao homem na sua totalidade, enquanto o treinamento seria específico e estaria voltado para a situação de trabalho. Goldstein (1991) definiu treinamento como "a aquisição sistemática de atitudes, conceitos, conhecimentos, regras ou habilidades que resultam no aumento da performance no trabalho" (p. 508). Também Latham (1988, p. 548) define treinamento como "o desenvolvimento sistemático de padrões de atitudes, conhecimentos e habilidades requeridos por um indivíduo para desenvolver adequadamente uma tarefa ou trabalho".

Treinamento tem sido apresentado nas organizações utilizando a abordagem sistêmica. O sistema de treinamento é composto dos seguintes elementos: identificação de necessidades de treinamento, planejamento do treinamento e avaliação do treinamento. Esse sistema é afetado por variáveis do contexto organizacional, como o clima e o suporte para transferência das habilidades 
adquiridas. Segundo Goldstein (1991, p. 514), programas de treinamento interagem com o sistema da organização e são diretamente afetados por ele, como as políticas corporativas de seleção e filosofia gerencial. Para esse autor, treinamentos devem ser previstos nas organizações após a identificação das necessidades, diagnosticadas em três níveis: análise organizacional (investigação se o treinamento é realmente necessário e se existe suporte para transferência das habilidades adquiridas); a análise de tarefas (identificação dos conhecimentos, habilidades e atitudes); e a análise pessoal (análise do desempenho).

O suporte organizacional tem sido relatado como crucial para a transferência das habilidades adquiridas em treinamento. De acordo com Latham (1988, p. 550 ), o suporte organizacional para treinamento ocorre na medida em que os objetivos de treinamento são ligados aos objetivos organizacionais, são adequados à estratégia organizacional e na extensão com que implicam o progresso do plano de negócio da organização. Para Kozlowski et al. (2000, p. 182), pesquisadores da área têm notado que os procedimentos adotados para a avaliação de necessidades de treinamento não oferecem uma ligação explícita entre os objetivos estratégicos organizacionais e as necessidades de treinamento. Percebe-se, portanto, que a identificação de necessidades de treinamento guarda estreita relação com a estratégia organizacional e com o suporte organizacional para transferência das habilidades adquiridas em treinamento.

\section{Avaliação de Treinamento}

A avaliação do treinamento fornece informações à organização com o objetivo de assegurar a manutenção, alteração ou rejeição do evento instrucional. Hamblin (1978) definiu a avaliação de treinamento como "o ato de julgar se o treinamento valeu ou não a pena, em termos de algum critério de valor, à luz da informação disponível" (p. 21). Goldstein (1991, p. 557) considerou a avaliação de treinamento como "uma coleta sistemática de informações descritivas e valorativas necessárias para tornar efetivas as decisões de treinamento, relacionadas à seleção, adoção, valor e modificação de várias atividades instrucionais".

A literatura aponta diversos modelos de avaliação de treinamento, porém as abordagens tradicionais adotam o modelo de Kirkpatrick, adaptado por Hamblin (1978, p. 30), que compreende a avaliação de reação, aprendizagem, comportamento no cargo, organização e valor definitivo, mantendo esses níveis um forte relacionamento positivo entre si. Kirkpatrick (como citado em Hamblin, 
1978, p. 30) aponta somente quatro níveis de avaliação: reações, aprendizado, comportamento no cargo e resultado. Esses níveis supõem uma relação causal entre eles, embora admita que a corrente poderá partir-se em qualquer um dos elos. Contudo pesquisas vêm demonstrando que não há relação significativa entre eles, uma vez que têm diferentes preditores de transferência de treinamento.

Paula (1992, p. 17), por exemplo, concluiu que o fato de as avaliações de reação serem favoráveis não garante a ocorrência de aprendizagem, como, também, aprendizagem não assegura mudanças do comportamento no cargo que, por sua vez, podem não produzir mudanças na organização ou não afetar o valor final. Para ela, existem outras variáveis do contexto organizacional que influenciam a transferência do treinamento para o trabalho. Borges-Andrade (2000, p. 2) considera que, embora a organização possa ter definidos os objetivos do nível de valor final, deles derivando-se os objetivos do nível seguinte até os do primeiro nível, conforme o modelo preconizado por Hamblin (1978), para alcançá-los seriam necessárias outras ações interferentes que não o treinamento.

Baldwin e Ford (1988, p. 64) asseguram que a transferência do treinamento abrange a generalização da habilidade adquirida para o contexto de trabalho e a sua manutenção a longo prazo. Eles propuseram um modelo de avaliação de treinamento, que compreende três aspectos: inputs do treinamento (características da clientela, desenho do treinamento e ambiente organizacional); outputs do treinamento (aprendizagem e retenção) e condições de transferência (generalização e manutenção). As condições para que a transferência do treinamento ocorra dependem das características da clientela, do projeto de treinamento e de variáveis do contexto organizacional. Tais variáveis vêm sendo largamente pesquisadas com a finalidade de encontrar preditores de transferência e impacto de treinamento no trabalho.

Para Abbad-Oc (1999, p. 26), o impacto do treinamento no trabalho compreende os conceitos de transferência do treinamento e de desempenho no trabalho. Segundo a autora, transferência de treinamento refere-se à aplicação correta, no trabalho, de conhecimentos, habilidades e atitudes adquiridas em treinamento, e impacto do treinamento no trabalho refere-se à influência exercida pelo treinamento sobre o desempenho do treinado. Essa autora investigou as relações entre as variáveis relativas ao indivíduo, ao treinamento, ao contexto organizacional, às reações ao treinamento e ao impacto do treinamento no trabalho e encontrou que as variáveis relacionadas ao apoio psicossocial à transferência explicaram mais do que $50 \%$ do impacto do treinamento no trabalho.

Tannenbaum e Yukl (1992, p. 420) definiram transferência de treinamento como 
"o grau com o qual treinandos efetivamente aplicam, no trabalho, os conhecimentos, as habilidades e atitudes adquiridas no contexto de treinamento". Royer (1979, p. 55) define transferência de treinamento como a extensão em que a aprendizagem de um treinamento contribui para a resolução de problemas ou para a aprendizagem de subseqüente evento instrucional. Ele considera duas classes de transferência de aprendizagem: a primeira ocorre quando um treinamento partilha estímulo comum com o evento da transferência e a segunda, como resultado do próprio processo de aprendizagem.

Segundo Kozlowski et al. (2000, p. 159), transferência de treinamento tem apenas duas distinções: transferência horizontal, que ocorre por meio de diferentes rumos ou contextos, no mesmo nível da tarefa executada pelo indivíduo; e a transferência vertical, que se refere ao link entre os resultados do treinamento no nível do indivíduo e no nível organizacional. Para eles, a ocorrência da transferência horizontal depende de efeitos contextuais ao nível de top-down, que se refere aos fatores organizacionais e do grupo de trabalho que exercem efeitos diretos para a aprendizagem e a transferência, enquanto que a transferência vertical se refere ao processo bottom-up, ou seja, à influência dos resultados do treinamento para o nível do grupo e para os resultados organizacionais. Para Abbad e Sallorenzo (2001, p. 33), existem fortes evidências empíricas demonstrando que uma nova habilidade não é transferida para o trabalho por falta de apoio organizacional. Elas asseguram que variáveis do ambiente organizacional controlam os efeitos de treinamento e de quaisquer outras formas de aprendizagem. Portanto encontrar e controlar essas variáveis torna-se essencial para a eficácia do treinamento nas organizações.

\section{Delimitação do Problema de Pesquisa}

Partindo do pressuposto de que o suporte organizacional é a variável que mais prediz impacto do treinamento no trabalho, supõe-se que ele ocorra com maior intensidade quando as habilidades ensinadas forem relevantes para os objetivos estratégicos da organização. Com tal suposição, este estudo pesquisou as relações entre a relevância dos treinamentos para os objetivos estratégicos organizacionais, o suporte à transferência (Fatores Situacionais de Apoio, Suporte Material e Consequiências Associadas ao Uso das Novas Habilidades), as características da clientela (Idade, Sexo, Nível de Escolaridade, Cargo/Função exercido na empresa, Lotação e Tempo de Serviço na empresa) e o impacto do treinamento no trabalho. 


\section{Organização Estudada: Centrais Elétricas do Norte do Brasil S.A. - Eletronorte}

O estudo foi realizado na Centrais Elétricas do Norte do Brasil S.A - Eletronorte, subsidiária da Centrais Elétricas do Brasil S.A - Eletrobrás, com atuação nos estados da Região Norte e nos Estados de Mato Grosso e Tocantins, na Região Centro-Oeste, e no estado do Maranhão, na Região Nordeste, cujo negócio é a geração e transmissão de energia elétrica.

\section{Método, Instrumentos e Procedimentos de Coleta de Dados}

Três variáveis independentes foram objetos do estudo: Relevância do Treinamento para os Objetivos Estratégicos da Organização, Suporte à Transferência (Fatores Situacionais de Apoio, Suporte Material e Conseqüências Associadas ao Uso das Novas Habilidades) e Características Demográficas da Clientela (Idade, Sexo, Nível de Escolaridade, Cargo/Função, Lotação e Tempo de Serviço na Organização). A variável dependente foi o Impacto do Treinamento no Trabalho.

A análise da relevância do treinamento para os objetivos estratégicos organizacionais, segundo a percepção dos gestores, constituiu a primeira variável. A segunda variável compreendeu a opinião dos treinados sobre o suporte ambiental ao uso eficaz das habilidades adquiridas. Essa variável abrangeu dois fatores: Suporte Gerencial e Social (apoio gerencial, social e organizacional) e Suporte Material (qualidade, quantidade e disponibilidade de recursos materiais e financeiros e adequação do local de trabalho à transferência do treinamento). A variável características da clientela referiu-se às informações demográficas e funcionais dos treinados. A variável impacto do treinamento no trabalho exprimiu a auto-avaliação dos treinados acerca do seu desempenho, motivação, autoconfiança e abertura a mudanças no trabalho em decorrência do treinamento.

A primeira variável, avaliação da relevância dos treinamentos para os objetivos estratégicos organizacionais, foi obtida por meio de entrevistas estruturadas, com a utilização de um questionário com perguntas fechadas e uma aberta, aplicado numa amostra de 33 gestores da empresa. Essa avaliação permitiu a obtenção da percepção dos gestores sobre a relevância dos treinamentos realizados na empresa para os objetivos estratégicos da organização. A variável relevância foi definida neste estudo como a opinião dos gestores sobre a importância de cada treinamento para a consecução dos objetivos estratégicos da empresa. 
Essa variável foi medida por intermédio de um questionário contendo uma listagem de 22 treinamentos, associada a uma escala de 6 pontos, onde 1 equivale a irrelevante e 6 a essencial. O questionário continha duas relações dos treinamentos realizados na empresa, que formavam os dois grupos pesquisados: Grupo A (composto de 11 treinamentos cujas competências individuais ensinadas eram similares às competências corporativas requeridas pelos objetivos estratégicos da empresa) e Grupo B (composto de 11 treinamentos cujas competências individuais ensinadas não eram similares às competências corporativas requeridas pelos objetivos estratégicos da empresa).

Os entrevistados indicaram no questionário o grau de relevância do treinamento para os objetivos estratégicos da empresa, e era necessário $80 \%$ de acordo para essa afirmação ser considerada válida, conforme critério científico adotado (Pasqualli, 1997, p. 97). O questionário continha ainda uma questão aberta, em que o entrevistado fazia comentários sobre a razão da irrelevância ou da relevância dos treinamentos para os objetivos estratégicos da empresa. Na parte final do questionário constavam itens referentes aos dados demográficos e funcionais do entrevistado.

As variáveis Impacto do Treinamento no Trabalho e Suporte à Transferência de Treinamento foram medidas por meio de uma pesquisa de campo com 375 treinados. Para medir a variável Impacto do Treinamento no Trabalho foi utilizada a escala validada por Abbad-Oc (1999); e para medir a variável Suporte à Transferência, a escala validada por Abbad e Sallorenzo (2001). O questionário Impacto do Treinamento no Trabalho destinou-se à avaliação do impacto do treinamento nas atividades desenvolvidas pelo treinado, bem como do suporte à transferência (gerencial, social e material) proporcionado pela organização. É constituído de quatro partes: (1) Impacto do Treinamento no Trabalho; (2) Fatores Situacionais de Apoio; (3) Suporte Material; e (4) Conseqüências Associadas ao Uso das Novas Habilidades. As três últimas pertencem à escala de Suporte à Transferência. Os 12 itens da primeira parte do questionário, denominada Avaliação do Impacto do Treinamento no Trabalho estão associados a uma escala Likert de cinco pontos, cuja pontuação corresponde ao seguinte: 1 (discordo totalmente da afirmativa); 2 (discordo um pouco da afirmativa); 3 (não concordo nem discordo); 4 (concordo com a afirmativa) e 5 (concordo totalmente com a afirmativa). Os itens foram submetidos à validação estatística neste estudo por meio de análises fatoriais e análises de confiabilidade (Alphas de Cronbach) e revelou-se com alto índice de confiabilidade, de 0,90 para 367 casos válidos.

Os 22 itens de Suporte à Transferência encontram-se agrupados conforme 
três categorias de conteúdo: Fatores Situacionais de Apoio (nove itens), permite ao participante avaliar o apoio gerencial e social (do grupo de trabalho) ou organizacional à transferência do treinamento; Suporte Material (seis itens), refere-se à qualidade, suficiência e disponibilidade de recursos materiais e financeiros, bem como à adequação do ambiente físico do local de trabalho à transferência do treinamento; e Consequiências Associadas ao Uso de Novas Habilidades (sete itens), refere-se à opinião do treinado sobre ocorrência de reações, favoráveis ou desfavoráveis, dos colegas, pares ou superiores à transferência do treinamento.

Todos os itens estão associados a uma escala de cinco pontos, com as seguintes correspondências: 1 (nunca); 2 (raramente); 3 (algumas vezes); 4 (freqüentemente); e 5 (sempre). Foram adotados os mesmos procedimentos de validação estatística utilizados na escala anterior. Encontraram-se dois fatores correlacionados entre si: Fator 1: Suporte Gerencial e Social à Transferência de Treinamento com 356 casos válidos e Fator 2: Suporte Material à Transferência de Treinamento, com 368 casos válidos, com Alphas de Cronbach de 0,91 e 0,86 respectivamente.

\section{Amostra de Participantes}

Foram entrevistados 33 gestores na pesquisa, cujas características são as seguintes. A maioria tem idade entre 48 e 53 anos, correspondendo a 78,8\%, predominando o sexo masculino, com 97\%. A maioria tem nível de escolaridade elevada, 48,5\% tem especialização ou MBA, 24,2\% tem mestrado e 3\% tem doutorado. Com relação aos dados funcionais, a maioria ocupa o primeiro e o segundo escalão da empresa; $3 \%$ ocupa cargo de diretor; 42,4\%, de superintendente; e $36,4 \%$, de assistente de diretor. Isso significa que $81,8 \%$ da amostra de gestores pesquisados é responsável diretamente pela formulação do Plano Estratégico da Organização. Quanto à lotação, 87,9\% dos entrevistados estão lotados na sede da organização e 12,1\% são lotados nas unidades descentralizadas. No que se refere ao tempo de serviço, a maioria tem mais de 21 anos na empresa, correspondendo a $66,7 \%$ da amostra, o que demonstra que esse grupo possui sólidos conhecimentos da empresa.

Quanto aos empregados treinados do total de 1200 participações em treinamento, 375 responderam à pesquisa, 196 referentes aos treinamentos do grupo $\mathrm{A}, \mathrm{e} 179$ referentes aos treinamentos do grupo B, o que correspondeu a 31,2\% do universo. A maioria tem entre 42 e 47 anos, equivalente a 41,6\% da amostra, seguida dos que têm entre 36 e 41 anos, que correspondem a 26,6\%, e dos que têm entre 48 
e 53 anos, o equivalente a 19,7\%. Tais índices, aliados ao fato de 2,2\% dos empregados treinados terem entre 54 e 59 anos, significa que $63,5 \%$ dos participantes dos treinamentos pesquisados na organização têm entre 42 e 59 anos, enquanto apenas 36,5\% têm entre 18 e 41 anos.

Quanto ao gênero dos treinandos, constata-se que a maioria é do sexo masculino, $65,3 \%$, e apenas $34,7 \%$, do sexo feminino. No que se refere ao nível de escolaridade, verifica-se que a maioria dos treinados tem especialização ou MBA, correspondendo a $32,9 \%$; em seguida vêm os que têm o $3^{\circ}$ grau completo $(28,8 \%)$. Apenas $0,9 \%$ possui o $2^{\circ}$ grau incompleto e $0,3 \%$, o $1^{\circ}$ grau incompleto. Quanto a cargo/função exercido na empresa, constata-se que o maior número de treinados foi o de nível médio, equivalente a $40,9 \%$, seguido do técnico de nível superior, $38,1 \%$, do gerente-executivo, $10,7 \%$, do superintendente, equivalente a $6,9 \%$, e do gerente de setor, $3,5 \%$ do total. Com relação à lotação dos participantes de treinamento, constata-se que 51,9\% são lotados na sede da empresa e 48,1\% estão lotados nas unidades descentralizadas.

No que se refere ao tempo de serviço na empresa, observa-se que $34,2 \%$ dos treinados têm de 11 a 15 anos de empresa, seguidos de $33,2 \%$ dos treinados que têm de 16 a 20 anos de empresa, $22,9 \%$ dos treinados que têm entre 21 e 25 anos, 5,3\% dos treinados que têm acima de 25 anos de empresa, $3,1 \%$ que têm até cinco anos de empresa e de $1,3 \%$ dos treinados que têm entre 6 e 10 anos de empresa.

\section{Resultados Encontrados}

Na análise da variável Relevância do Treinamento para os Objetivos Estratégicos da Organização, encontrou-se que a maioria dos gestores pesquisados avalia os treinamentos na categoria essencial (compreendendo os pontos 6, 5 e 4) na escala do instrumento. Na categoria irrelevante, (compreendendo aos pontos 1, 2 e 3), observou-se que houve um número menor de avaliações. Isso significa que os gestores consideraram a maioria dos treinamentos relevantes para os objetivos estratégicos da organização, em maior ou menor grau.

A Tabela 1 apresenta a avaliação dos gestores conforme a escala original do instrumento, referente aos treinamentos do grupo A, e a Tabela 2, a mesma avaliação para os do grupo B. 
Tabela 1: Frequiências da Variável Relevância do Treinamento para os Objetivos Estratégicos da Eletronorte na Escala Original (Grupo A)

\begin{tabular}{|c|c|c|c|c|c|c|}
\hline \multirow{2}{*}{ TREINAMENTO } & \multicolumn{6}{|c|}{ FREQÜÊNCIA RELATIVA NA ESCALA ORIGINAL } \\
\hline & 1 & 2 & 3 & 4 & 5 & 6 \\
\hline $\mathrm{R} / 3$ SIN- Sistema Integrado de Gestão & - & - & - & - & $27,3 \%$ & $72,7 \%$ \\
\hline Preparação para a banca PNQ & - & - & $3 \%$ & $27,3 \%$ & $42,4 \%$ & $27,3 \%$ \\
\hline Preparação para a banca PQGF & $3 \%$ & - & $9,1 \%$ & $39,4 \%$ & $36,4 \%$ & $12,1 \%$ \\
\hline ISO 14000 - Auditor Ambiental & - & $3 \%$ & - & $24,2 \%$ & $36,4 \%$ & $36,4 \%$ \\
\hline TPM - Manutenção Produtiva Total & - & - & - & $6,1 \%$ & $36,4 \%$ & $57,6 \%$ \\
\hline MBA - Gestão Empresarial & - & - & $9,1 \%$ & $24,2 \%$ & $36,4 \%$ & $30,3 \%$ \\
\hline Regras de Mercado & - & - & $6,1 \%$ & $9,1 \%$ & $21,2 \%$ & $63,6 \%$ \\
\hline $\begin{array}{l}\text { Planos de Contingências e Emergências } \\
\text { Ambientais }\end{array}$ & - & - & $3 \%$ & $39,4 \%$ & $33,3 \%$ & $24,2 \%$ \\
\hline $\begin{array}{l}\text { Custos Marginais e Tarifação de Energia } \\
\text { Elétrica }\end{array}$ & - & $3 \%$ & $\begin{array}{r}12,1 \\
\%\end{array}$ & $9,1 \%$ & $30,3 \%$ & $45,5 \%$ \\
\hline $\begin{array}{l}\text { Seminário Internacional de Transmissão } \\
\text { de Energia Elétrica }\end{array}$ & $3 \%$ & $9,1 \%$ & $6,1 \%$ & $39,4 \%$ & $21,2 \%$ & $21,2 \%$ \\
\hline $\begin{array}{l}\text { Estratégia de Negociação e Conflitos } \\
\text { Ambientais }\end{array}$ & - & $6,1 \%$ & $9,1 \%$ & $27,3 \%$ & $36,4 \%$ & $21,2 \%$ \\
\hline
\end{tabular}

\section{Tabela 2: Freqüiências da Variável Relevância do Treinamento para os Objetivos Estratégicos da Eletronorte na Escala Original (Grupo B)}

\begin{tabular}{l|r|r|r|r|r|r}
\hline \multirow{2}{*}{ TrEINAMENTO } & \multicolumn{5}{c}{ FREQÜÊNCIA RELATIVA NA ESCALA ORIGINAL } \\
\cline { 2 - 7 } & $\mathbf{1}$ & $\mathbf{2}$ & $\mathbf{3}$ & $\mathbf{4}$ & $\mathbf{5}$ & \multicolumn{1}{c}{$\mathbf{6}$} \\
\hline ACS - Atualização de Chefes e & - & $3 \%$ & $12,1 \%$ & $24,2 \%$ & $36,4 \%$ & $24,2 \%$ \\
Supervisores & $3 \%$ & $9,1 \%$ & $18,2 \%$ & $42,4 \%$ & $27,3 \%$ & - \\
MBA - Auditoria & - & $6,1 \%$ & $9,1 \%$ & $30,3 \%$ & $36,4 \%$ & $18,2 \%$ \\
MBA - Meio Ambiente & - & $6,3 \%$ & $3,1 \%$ & $28,1 \%$ & $34,4 \%$ & $28,1 \%$ \\
MBA - Gestão de Pessoas por & & & & & & \\
Competências & $6,1 \%$ & $6,1 \%$ & $33,3 \%$ & $24,2 \%$ & $18,2 \%$ & $12,1 \%$ \\
Preposto (Representação Jurídica da & $3,2 \%$ & $3,2 \%$ & $19,4 \%$ & $35,5 \%$ & $25,8 \%$ & $12,9 \%$ \\
Empresa) & $3 \%$ & - & $21,2 \%$ & $45,5 \%$ & $24,2 \%$ & $6,1 \%$ \\
Anatem (Informática) & - & $6,1 \%$ & $18,2 \%$ & $27,3 \%$ & $15,2 \%$ & $33,3 \%$ \\
Inglês & - & $6,3 \%$ & $28,1 \%$ & $34,4 \%$ & $15,6 \%$ & $15,6 \%$ \\
Informática (Word, Windows etc.) & $9,1 \%$ & $15,2 \%$ & $21,2 \%$ & $27,3 \%$ & $24,2 \%$ & $3 \%$ \\
Primeiros Socorros & & $12,5 \%$ & $21,9 \%$ & $25 \%$ & $31,3 \%$ & $9,4 \%$ \\
Projeto Bem-Viver (Ikebana, Tai-Chi- & - & & & & \\
Chuan) & Microscada (Treinamento Contratual) & - & &
\end{tabular}

Nas análises estatísticas, utilizou-se o Teste T para verificar as diferenças entre as médias das variáveis Impacto de Treinamento no Trabalho e Suporte à Transferência, compreendendo duas variáveis independentes, Suporte Gerencial e Social à Transferência de Treinamento (fatores situacionais de apoio e conseqüências 
associadas ao uso das novas habilidades); e Suporte Material à Transferência, referentes a cada grupo de treinamento. Os resultados encontrados demonstraram existirem diferenças significativas entre as médias das variáveis nos dois grupos de treinamento com $t=2,780$ e nível de significância de $p<0,01$ para a variável Impacto do Treinamento no Trabalho; $\mathrm{t}=3,470$ e $\mathrm{p}<0,01$ para a variável Suporte Gerencial e Social à Transferência e $\mathrm{t}=4,317$ e $\mathrm{p}<0,001$ para a variável Suporte Material à Transferência. A Tabela 3 apresenta as médias e o desvio padrão de cada grupo de treinamento e a Tabela 4, os valores de t e níveis de significância.

\section{Tabela 3: T-teste das Médias e Desvios Padrões por Grupo de Treinamento}

\begin{tabular}{lccccc}
\hline \multicolumn{1}{c|}{ VARIÁVEIS } & $\begin{array}{c}\text { GRUPO DE } \\
\text { TREINAMENTO }\end{array}$ & $\begin{array}{c}\text { NÚMERO DE } \\
\text { RESPONDENTES }\end{array}$ & MÉDIA & DESVIO PADRÃo \\
\hline $\begin{array}{l}\text { Impacto de } \\
\text { treinamento no }\end{array}$ & $\mathrm{A}$ & 197 & 4,02 & 0,64 \\
trabalho & $\mathrm{B}$ & 178 & 3,81 & 0,81 \\
$\begin{array}{l}\text { Suporte gerencial e } \\
\text { social à } \\
\text { transferência }\end{array}$ & $\mathrm{A}$ & 197 & 3,31 & 0,72 \\
$\begin{array}{l}\text { Suporte material à } \\
\text { transferência }\end{array}$ & $\mathrm{B}$ & 178 & 3,08 & 0,82 \\
\hline
\end{tabular}

Tabela 4: T-teste de Igualdade das Médias dos Grupos de Treinamento

\begin{tabular}{l|c|l}
\hline \multicolumn{1}{c|}{ VARIÁVEIS } & T & NÍVEL DE SIGNIFICÂNCIA \\
\hline Impacto de treinamento no trabalho & 2,780 & $\mathrm{p}=0,006<0,01$ \\
Suporte gerencial e social à transferência & 3,470 & $\mathrm{p}=0,001<0,01$ \\
Suporte material à transferência & 4,317 & $\mathrm{p}=0,0001<0,001$ \\
\hline
\end{tabular}

Pelos níveis de significância encontrados nas diferenças entre as variáveis Impacto de Treinamento no Trabalho, Suporte Gerencial e Social e Suporte Material à transferência e os grupos de treinamento A e B, conclui-se que são significativos para confirmar a hipótese dessa pesquisa. Observa-se que Suporte Gerencial e Social e Suporte Material têm nível de significância menor que o de impacto de treinamento no trabalho, demonstrando que o suporte organizacional à transferência é maior, quando os treinamentos são relevantes para a estratégia organizacional.

$\mathrm{Na}$ análise de regressão, utilizou-se o método de regressão padrão, tendo em vista que a pesquisa teve caráter exploratório e a amostra foi pequena. Foram considerados 356 casos válidos da amostra após a retirada dos outliers. Na primeira equação da regressão padrão foram utilizadas todas as variáveis independentes do 
estudo para investigar a relação de predição sobre a variável dependente Impacto do Treinamento no Trabalho. São elas: Suporte à Transferência, compreendendo duas variáveis independentes, Suporte Gerencial e Social à Transferência de Treinamento (fatores situacionais de apoio e consequiências associadas ao uso das novas habilidades) e Suporte Material à Transferência de Treinamento; variáveis demográficas (idade, sexo, nível de escolaridade, cargo/função exercido atualmente na Eletronorte, tempo de serviço na Eletronorte) e a variável Grupos de Treinamento A e B. A variável lotação teve um número maior que $5 \%$ de valores omissos e foi retirada da análise. Nessa análise, o $\mathrm{R}^{2}$ ajustado encontrado foi de 0,37, com nível de significância $\mathrm{p}<0,0001$.

A variável Suporte Gerencial e Social à Transferência de Treinamento apresentou um nível de significância $\mathrm{p}<0,0001$, indicando que esta variável é a principal preditora de impacto de treinamento no trabalho na organização. Entre todas as variáveis antecedentes, suporte gerencial e social à transferência foi a maior preditora de impacto de treinamento no trabalho na avaliação dos treinados com $\mathrm{Sr}^{2}$ 0,53, com Beta de 0,58. O Beta desta variável indica que, quanto maior a percepção de Suporte Gerencial e Social pelos treinados, maior a percepção de Impacto do Treinamento no Trabalho. Entre as variáveis demográficas, foram significativas na predição de impacto de treinamento no trabalho, $\mathrm{p}<0,05$, escolaridade ( $1^{\circ}$ grau incompleto), com Beta negativo, e cargo (empregado de nível médio), com Beta positivo. Isso significa que para os empregados com escolaridade mais baixa a ocorrência do impacto do treinamento no trabalho é menor, enquanto para os empregados de nível médio é maior. A Tabela 5 apresenta as correlações entre as variáveis, os coeficientes de regressão não-padronizados (B), a constante, os coeficientes de regressão padronizados $(\beta)$, as correlações semiparciais $\left(\mathrm{Sr}^{2}\right)$, o $\mathrm{R}$, o $\mathrm{R}^{2}$ e o $\mathrm{R}^{2}$ ajustado.

\section{Tabela 5: Primeira Regressão Padrão na Predição do Impacto do Treinamento no Trabalho com Todas as Variáveis Independentes}

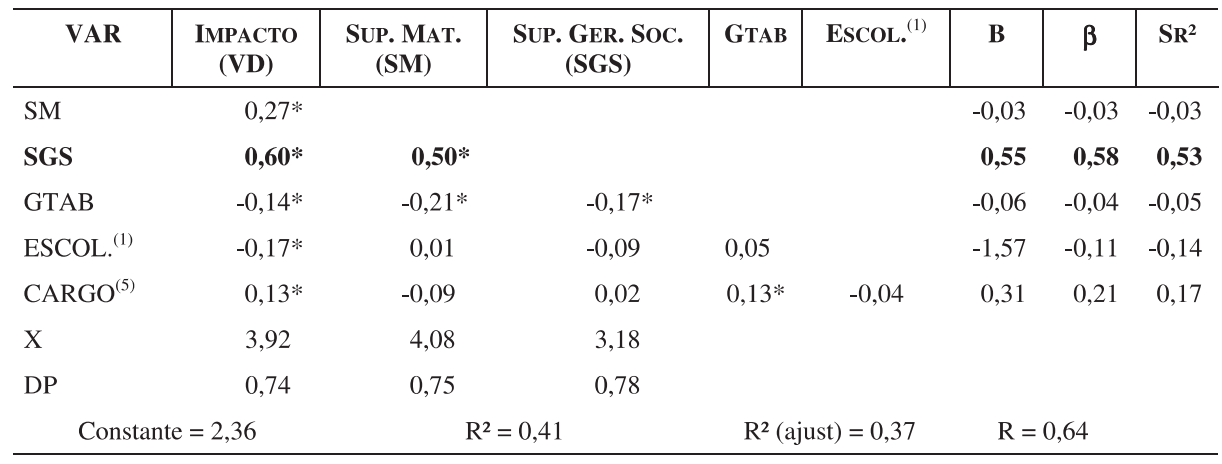

Nota: $* \mathrm{p}<=0,001 \quad$ (1) ESCOL. $=1^{\circ}$ Grau incompleto (5) CARGO = Empregado de nível médio 
A segunda análise de regressão padrão, tendo como variáveis independentes somente as variáveis demográficas e grupos de treinamento A e B, apresentou os seguintes resultados: $\mathrm{R}^{2}$ ajustado de 0,06 com nível de significância $\mathrm{p}<0,05$. As variáveis demográficas significativas foram escolaridade ( $\left(1^{\circ} \mathrm{Grau}\right.$ incompleto $)$ e ( $3^{\circ}$ Grau completo), ambas com Beta negativo, indicando que, para os empregados enquadrados nessas categorias, a percepção de impacto do treinamento no trabalho foi menor e cargo ocupado (empregado de nível médio), com Beta positivo, indicando que, para os empregados que ocupam esse cargo, houve maior percepção de impacto do treinamento no trabalho. Nessa análise, os grupos de treinamento A e B também foram significativos, com Beta negativo, indicando que os treinamentos do grupo A tem maior impacto. A Tabela 6 apresenta as correlações entre as variáveis, os coeficientes de regressão não-padronizados (B), a constante, os coeficientes de regressão padronizados $(\beta)$, as correlações semiparciais $\left(\mathrm{Sr}^{2}\right)$, o $\mathrm{R}$, o $\mathrm{R}^{2}$ e o $\mathrm{R}^{2}$ ajustado.

\section{Tabela 6: Segunda Regressão Padrão na Predição do Impacto do Treinamento no Trabalho com as Variáveis Demográficas e Grupos de Treinamento como Variáveis Independentes}

\begin{tabular}{|c|c|c|c|c|c|c|c|}
\hline VAR & $\begin{array}{c}\text { IMPACTO } \\
\text { (VD) }\end{array}$ & GTAB & ESCOL. ${ }^{(1)}$ & ESCOL. ${ }^{(5)}$ & B & $\beta$ & $\mathbf{S R}^{2}$ \\
\hline GTAB & \multicolumn{3}{|l|}{$-0,14 *$} & \multicolumn{2}{|r|}{$-0,19$} & $-0,12$ & $-0,12$ \\
\hline ESCOL. ${ }^{(1)}$ & $-0,17 *$ & \multicolumn{2}{|l|}{0,05} & \multicolumn{2}{|r|}{$-2,32$} & $-0,16$ & $-0,17$ \\
\hline ESCOL. ${ }^{(5)}$ & $-0,04$ & 0,001 & $-0,01$ & \multicolumn{2}{|r|}{$-0,35$} & $-0,14$ & $-0,12$ \\
\hline $\mathrm{CARGO}^{(5)}$ & $0,13 *$ & $0,13 *$ & $-0,04$ & $0,36^{*}$ & \multirow[t]{2}{*}{0,44} & \multirow[t]{2}{*}{0,29} & 0,19 \\
\hline $\mathrm{X}$ & 3,92 & & & & & & \\
\hline DP & 0,74 & & & & & & \\
\hline \multicolumn{2}{|c|}{ Constante $=4,03$} & \multicolumn{2}{|c|}{$\mathrm{R}^{2}=0,12$} & \multicolumn{2}{|c|}{$\mathrm{R}^{2}$ (ajust) $=0,06$} & \multicolumn{2}{|c|}{$\mathrm{R}=0,35$} \\
\hline
\end{tabular}

Nota: $* \mathrm{p}<=0,001$ (1) ESCOL. $=1^{\circ}$ Grau incompleto (5) ESCOL. $=3^{\circ}$ Grau completo (5) $\mathrm{CARGO}=$ Empregado de nível médio.

\section{CONCLUSÃo}

Os resultados encontrados confirmam a existência de diferenças significativas entre as variáveis: Impacto do Treinamento no Trabalho e Suporte à Transferência de Treinamento (Suporte Gerencial e Social e Suporte Material), para os grupos de treinamento A (treinamentos cujas competências individuais ensinadas são similares às competências corporativas requeridas pelos objetivos estratégicos organizacionais) e B (treinamentos cujas competências individuais ensinadas não são similares às competências corporativas requeridas pelos objetivos estratégicos 
organizacionais). Portanto, o impacto do treinamento no trabalho é maior, quando os treinamentos são relevantes para os objetivos estratégicos organizacionais, confirmando a hipótese desta pesquisa.

O Suporte Gerencial e Social mostrou-se a mais forte variável preditora de impacto do treinamento no trabalho, corroborando os resultados encontrados na literatura, que demonstraram ser o suporte psicossocial à transferência o maior preditor de impacto de treinamento no trabalho (Abbad-Oc, 1999; Meneses, 2002; Rodrigues, 2000; Sallorenzo, 2000; Tamayo, 2002).

A variável Relevância do Treinamento para os Objetivos Estratégicos Organizacionais teve uma contribuição pequena, porém significativa, na predição do Impacto do Treinamento no Trabalho. Isso aponta a importância de as organizações realizarem os treinamentos considerando os objetivos estratégicos organizacionais. Esse achado vem ao encontro do recente surgimento das Universidades Corporativas, cujo propósito é estabelecer programas de treinamento alinhados ao planejamento empresarial.

A implicação teórica do estudo é a ratificação da necessidade de os modelos de avaliação de treinamento incluírem metodologias de mensuração do impacto do treinamento não só no nível individual, mas também no nível das equipes e dos objetivos organizacionais, conforme preconiza a abordagem multinível de Kozlowski et al. (2000, p. 159).

A pesquisa pretendeu acender uma pequena luz nessa direção, na medida em que investigou as variáveis do contexto organizacional necessárias para a transferência individual, incorporando uma variável organizacional: a relevância do treinamento para os objetivos estratégicos da organização, na tentativa de encontrar um elo comum entre essas variáveis para a predição do impacto do treinamento no trabalho.

Os resultados apontam uma possível interação do impacto do treinamento no trabalho do indivíduo com o impacto no nível organizacional. Além disso, acrescenta-se uma discussão teórica sobre as razões pelas quais os indivíduos transferem para o trabalho as habilidades adquiridas em treinamento e as expandem para o contexto organizacional, quando percebem a relevância do treinamento para a organização.

A hipótese da pesquisa foi confirmada juntamente com as demais variáveis independentes que vêm sendo pesquisadas na literatura para a predição do impacto do treinamento no trabalho. Dessa forma, pesquisas devem continuar investigando evidências que comprovem se treinamentos com objetivos relevantes para os 
objetivos estratégicos da organização têm maior impacto no trabalho do indivíduo, e se esse impacto se expande para o nível do grupo e da organização.

\section{Artigo recebido em 20.05.2004. Aprovado em 23.09.2004.}

\section{ReferênCIAS Bibliográficas}

Abbad-Oc, G. (1999).

Um modelo integrado de avaliação do impacto do treinamento no trabalho - IMPACT. Tese de Doutoramento não-publicada, Instituto de Psicologia, Universidade de Brasília, DF, Brasil.

Abbad, G., \&

Sallorenzo, L. H. (2001, abril/junho).

Desenvolvimento e validação de escalas de suporte à transferência de treinamento. Revista de Administração da USP, 36(2), 33-45.

Baldwin, T., \&

Ford, J. K. (1988).

Transfer of training: a review and directions for future research. Personnel Psychology, 41(1), 63-105.

Bastos, A. V. B. (1991, outubro/ dezembro).

O suporte oferecido pela pesquisa na área de treinamento. Revista de Administração, 26(4), 87-102.

Borges-Andrade, J. E. (2000).

Desenvolvimento de medidas em avaliação de treinamentos. Anais do Encontro Nacional dos Programas de Pós-Graduação em Administração, Florianópolis, SC, 24.
Campbell, J. P. (1988).

Training Design for Performance Improvement. In J. P. Campbell., \& R. J. Campbell, Productivity in organizations (pp.177-215). San Francisco: Jossey-Bass.

Goldstein, I. L. (1991).

Training in work organizations. In Dunnette e Hough Handbook of Industrial and Organizational Psychology (2nd ed., pp.507-619). Califórnia: Consulting Psychology Press.

Green, P. C. (1999).

Building robust competencies. San Francisco: Jossey-Bass, 1999.

Hamblin, A. C. (1978).

Avaliação e controle de treinamento. São Paulo: McGraw-Hill.

Hamel, G., \&

Prahalad, C. K. (1995).

Competindo pelo futuro: estratégias inovadoras para obter o controle do seu setor e criar os mercados de amanhã. Rio de Janeiro: Campus.

Hill, C., \&

Jones, G. (1998).

Strategic management: an integrated approach (4th ed.). Boston: Houghton Mifflin Company. 
Kamoche, K. (1996, March).

Strategic Human Resource Management within a ResourceCapability View of the Firm. Journal of Management Studies, 33(2), 213233.

Kozlowski, S. W. J.,

Brown K. G.,

Weissbein D. A.,

Cannon-Bowers J. A., \&

Salas, E. (2000).

A Multilevel approach to training effectiveness: enhancing horizontal and vertical transfer. In K. J., Klein, S. W. J. Kozlowski, Multilevel theory, research, and methods in organizations: foundations, extensions, and new directions (pp. 157-210). San Francisco: JosseyBass.

Latham, G. P. (1988, January).

Human resource training and development. Annual Review of Psychology, 39, 545-582.

Legge, K. (1995).

Human resource management: rhetorics and realities. London: Macmillan Press.

Lengnick-Hall, C. A., \&

Lengnick-Hall, M. L. (1990).

Interactive human resource management and strategic planning. Westport: Quorum Books.

Meneses, P. P. M. (2002).

Auto-eficácia, locus de controle, suporte à transferência e impacto do treinamento no trabalho. Dissertação de Mestrado nãopublicada, Instituto de Psicologia, Universidade de Brasília, DF, Brasil.
Nadler, L. (1984).

The handbook of human resource development. New York: Wiley.

Oliveira, M. M., Jr. (2001).

Competências Essenciais e Conhecimento na Empresa. In M. T. LFleury, \& M. M., Jr, Oliveira Gestão Estratégica do Conhecimento. São Paulo: Atlas.

Pasquali, L. (1997).

Psicometria: Teoria e Aplicações. Brasília: UnB.

Paula, S. M. A. de (1992).

Variáveis preditoras de impacto de treinamento no trabalho: análise da percepção dos treinados de duas organizações. Dissertação de Mestrado não-publicada, Instituto de Psicologia, Universidade de Brasília, DF, Brasil.

Porter, M. E. (1989).

Vantagem competitiva (4a ed.). Rio de Janeiro: Campus.

Royer, J. M. (1979).

Theories of the transfer of learning. Educational Psychologist, 14, pp. 53-69.

Rodrigues, A. G. (2000).

A natureza da participação e suas implicações no impacto do treinamento no trabalho. Dissertação de Mestrado nãopublicada, Instituto de Psicologia, Universidade de Brasília, DF, Brasil.

Salas, E., \&

Cannon-Bowers, J. A. (2001).

The science of training: a decade of progress. Annual Review of Psychology, (52), pp. 471-499. 
Sallorenzo, L. H. (2000).

Avaliação de impacto de treinamento no trabalho: analisando e comparando modelos de predição. Dissertação de Mestrado não-publicada, Instituto de Psicologia, Universidade de Brasília, DF, Brasil.

Tamayo, N. (2002).

Autoconceito profissional, suporte à transferência e impacto do treinamento no trabalho. Dissertação de
Mestrado não-publicada, Instituto de Psicologia, Universidade de Brasília, DF, Brasil.

Tanenbaum, S. I., \& Yukl, G. (1992).

Training and development in work organizations. Annual Review of Psychology, 43, 399-441.

Ulrich, D. (1998).

Os campeões de recursos humanos (2nd ed.). São Paulo: Futura. 